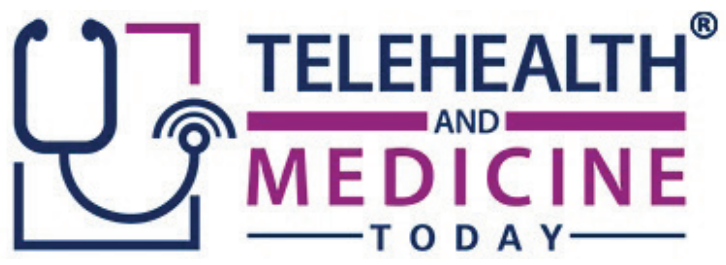

\title{
Implementation and Optimization of Telehealth during the COVID-19 Public Health Crisis-Our Journey
}

\section{Claude J. Pirtle, Anna Tetleton-Burns, Ashley Webb, Scott Krodel}

Affiliation: West Tennessee Healthcare, Jackson, TN, USA

Corresponding Author: Claude J. Pirtle, Email: claude.pirtlemd@wth.org

Keywords: Change Management, Rural Communities, Telehealth, Telemedicine

Section: Use Cases, Pilots

Telehealth offered a great opportunity for the clinicians at West Tennessee Healthcare to provide an essential service to the community at large during these unprecedented times. Our telehealth footprint continues to enlarge daily with more clinicians and specialties becoming comfortable with the workflows and technology. The program is described herein.

$\mathrm{T}$ The COVID-19 pandemic tests the ability of many primary care, urgent care, and hospital systems to adapt quickly to new circumstances. In many of these offices, virtual telehealth visits have become a strategy to decrease the use of personal protective equipment, and mitigate the risk of exposure of providers, staff, or patients to the coronavirus, among many other opportunities. ${ }^{1}$ West Tennessee Healthcare noted these same strong headwinds, as the pandemic and emergency decelerations began to send individuals to continue to reschedule appointments for a multitude of reasons - including fear.

West Tennessee Healthcare is located in an underserved area with a lack of access to healthcare in all of our servicing districts. Each of these districts holds a classification of a partial or full healthcare professional shortage area. In our over 40 primary and specialty care clinics, face-to-face visits began to shudder near the end of March with the Tennessee governor's first social distancing and business step-down regulations. With the foresight of other health systems being impacted by COVID-19, our medical practices were forced into a dilemma to creatively continue to offer quality care, but from a new environment. Prior to this federal and state emergency, our clinics were in the planning stages of creating a telehealth environment but had not completed any medical telehealth visits. 


\section{VISION DESIGN AND STRATEGY}

Our journey began knowing that we were in a very fluid period. Using principles of strategic design and planning, our team created a Telehealth Governance Council comprising clinicians, clinic directors, nursing, and administration. Our council chose to use an abbreviated version of Kotter's eight-step change management strategy ${ }^{2}$ (Figure 1) followed by a gap analysis.

Our vision was to have solutions built across the enterprise and appropriate for multiple electronic health record (EHR) platforms, including AthenaHealth (Athena), GE Centricity, Cerner, and eClinicalWorks. Ultimately, an integrated video solution was chosen with our AthenaHealth platform, and a third party agnostic solution was to be used for other EHR platforms. On March 17, 2020, the Office for Civil Rights at the US Department of Health and Human Services announced that it would "exercise its enforcement discretion and waive potential penalties for HIPAA (Health Insurance Portability and Accountability Act of 1996) violations against healthcare providers that serve patients through everyday communications technologies during the COVID-19 nationwide public emergency."3 Our group chose to use only HIPAA-secure solutions because of the understanding that

\begin{tabular}{|l|}
\hline \multicolumn{1}{|c|}{ Eight-step process for leading change } \\
\hline 1. Establish a sense of urgency \\
\hline 2. Create a guiding coalition \\
\hline 3. Develop a vision for change \\
\hline 4. Communicate the vision \\
\hline 5. Remove obstacles \\
\hline 6. Generate short-term wins \\
\hline 7. Sustain acceleration \\
\hline 8. Institute a permanent action \\
\hline
\end{tabular}

Figure 1-Kotter's eight-step change management strategy. this regulation would likely be rolled back, and to supply the most appropriate solution to ensure privacy protection to our patients.

\section{SOLUTION IMPLEMENTATION AND GAP ANALYSIS}

Our governing council decided to begin with a pilot with our integrated solution in the majority of our clinics due to perceived barriers using a separate option. The integration of the tool into the EHR allowed options such as streamlining the presentation of telehealth visits on the provider's schedule, and simplified workflows for nursing and scheduling, among many other small but significant wins.

The council chose to create a structure for only a portion of our primary care clinics and specialty clinics in an effort to solidify workflow and perform a gap analysis to optimize the experience for the clinician and patient. A standardized workflow was approved by the council and then tested with staff members to identify potential knowledge gaps.

\section{COMMUNICATION AND STANDARDIZATION}

The council created standard correspondence and disseminated it for each of the patients prior to their visit. Depending on whether the patients were established or new, they would receive the same type of information. This communication included instructions on how to install the application associated with their telehealth visit, current regulation and privacy policies, and also a structured diagram to allow quick support.

A script was crafted for each of the schedulers to follow when calling patients to schedule the visit, or if the patient were to call in to schedule a visit (Figure 2). Each clinic also sent out a standard letter to each of the patients via the patient portal 


\begin{tabular}{|l|}
\hline Telehealth scheduling workflow - Integrated Hale Health Solution - COVID-19 \\
\hline $\begin{array}{l}\text { 1. All established patients will be offered the scheduling the option of a telehealth visit based on } \\
\text { parameters discussed with providers in their office. Telehealth visits should be offered to } \\
\text { applicable patients calling to schedule/reschedule visits or those we reach out to. After } \\
\text { scheduling the telehealth visit, schedulers should remain on the line to ensure patients } \\
\text { receive the invite to the e-mail or cell on file. If they do not receive the appropriate } \\
\text { information, the scheduler should advise patients that someone from our dedicated } \\
\text { telehealth team will be calling them to assist with setting up their telehealth visit }\end{array}$ \\
\hline $\begin{array}{l}\text { 2. Patients scheduling telehealth visits for follow-up can be provided Hale cards for more } \\
\text { information. }\end{array}$ \\
\hline $\begin{array}{l}\text { 3. Patients who have already arrived to the clinics should not be considered for telehealth visits. } \\
\text { Telehealth only applies to patients accessing providers from locations outside our clinic. }\end{array}$ \\
4. Providers wanting to access telehealth visits outside normal business hours should only \\
do so for patients already registered with a Hale account. \\
5. A dedicated staff member will be proactively addressing patient syncing issues for upcoming \\
appointments as well as proactively reaching out to any patient not yet activated in the \\
system with an upcoming appointment. \\
are visible in AthenaHealth; if not, please use Hale Health; export functionality to \\
load into the AthenaHealth chart. \\
prior to the provider joining the visit. The nurse should document the information in an open \\
conversation is exported, and is available. We will continue to optimize the triage \\
customization of Hale Health.
\end{tabular}

Figure 2-The script crafted for each of the schedulers to follow when calling patients to schedule the visit, or if the patient were to call in to schedule a visit.

and e-mails about the new offering of telehealth. This piece only allowed historical members of our medical community to be notified about our upcoming new service line; however print, TV, web, and radio ads were created to inform new patients in the community.

\section{TRAINING AND CULTURE}

Knowing that the technical component was put in place, our team concluded that the behavioral component would offer the largest challenge to the success of the project. ${ }^{4}$ The clinicians were already very familiar with the EHR. Our ambulatory EHR allowed a telehealth solution to be integrated into the clinical workflow relatively quickly and efficiently. Clinicians were individually trained in either of the two ways: (1) One-on-one training with support personal, or (2) through pre-recorded video and training aids.

The same type of training model was used for schedulers, nursing staff, and other staff members assisting with the system. At this point, the decision was made to create a dedicated support line in case at any time during the day, one source of truth could be accessed to assist staff members.

Understanding that training of not only the clinician and staff was to occur but also that of the patient, in our standardized script, we built in descriptors to better define the opportunities (e.g., access to care) and some of the limitations 
of telehealth (e.g., limited physical exam) in an effort to ensure that the patient had a well-rounded view of the opportunities and boundaries of telehealth. The patient was also furnished with the same dedicated support line in case issues or questions surfaced.

Custom content was crafted to help clinicians with documentation and billing burdens of each visit. Our group created statements that could automatically be pulled into the documentation in an effort to improve efficiency. The group also created quick reference training aids to allow clinicians a "quick" view into what a telehealth visit required.

\section{ALIGNING WORKFLOW AND UPDATED REGULATORY STANDARDS}

After reviewing our face-to-face appointment workflow, we noted a number of gaps that would occur when using a telehealth encounter. We redesigned the nursing, scheduling, and clinician workflows. One challenge that came to light was the role of nursing during a Medicare Annual Wellness Exam (MAWV) at a telehealth visit. After the Centers for Medicare \& Medicaid Services (CMS) acknowledged that MAWV could be completed via telehealth, we developed custom content for patients to complete during electronic intake prior to a telehealth visit in a secure manner. This included optimization of drawing the MAWV clock in each exam. The goal was to implement a solution that not only minimized variation but also did not constrain the time of the nursing staff.

\section{VISIT TYPES}

In addition, it was decided to offer telehealth visits for routine follow-ups and refills initially to established patients. The goal of this effort was to keep healthy patients out of the waiting rooms but still offer the same level of care as if they were seen in the clinic. Clinicians were also offered to review their schedule for individuals who could be seen via our telehealth solution. Following the first burst of patient visits, the scheduling staff was asked to offer telehealth visits first if the future visit was deemed appropriate. We did restrict the prescription of controlled substance refills other than refilling attention deficit/hyperactivity disorder (ADHD) and medications (e.g., lisdexamfetamine, Vyvanse) for established individuals with the caveat that they met the appropriate criteria and guidelines were followed. Following CMS's approval of new patients being seen via telehealth, we also began scheduling visits; however, this was a minority of the visits scheduled.

\section{MEASUREMENTS OF SUCCESS}

In 2019, during the March 23-28 time frame, the clinics using the integrated solution completed 2,183 evaluation and management (E\&M) visits. This year (during the beginning of the COVID crisis) these clinics completed 2,469 visits, including 71 telehealth visits. By week 4 of March, a total of 941 telehealth visits were completed with 195 of the visits being completed on week 4 . A total of 2,115 visits were completed in week 4 of March and 2,462 E\&M-coded visits from the same time period of the year prior (Figure 3 ). This constitutes an increase in $1300 \%$ over 4 weeks (Figure 4 ) in a portion of our ambulatory telehealth visits. The number of providers in this cohort increased by $15 \%$ in 2020 .

\section{CHALLENGES, LIMITATIONS, AND FEEDBACK}

Telehealth offers a great opportunity to continue high-quality care in a convenient manner. With the declaration of the emergency, many of the requirements, including originating sites and reimbursement requirements, have 


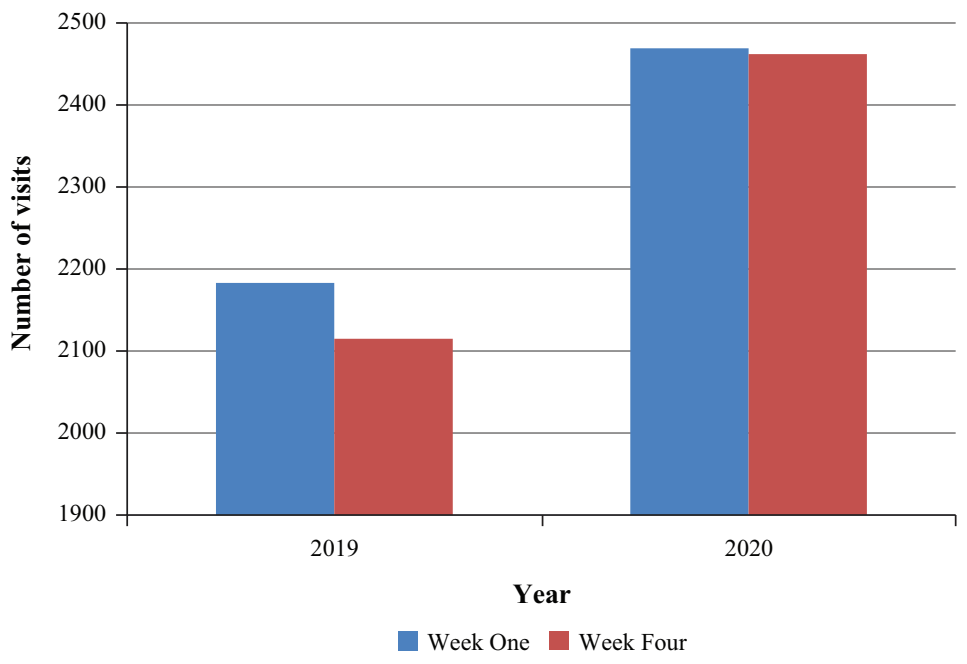

Figure 3-Ambulatory visits in March.

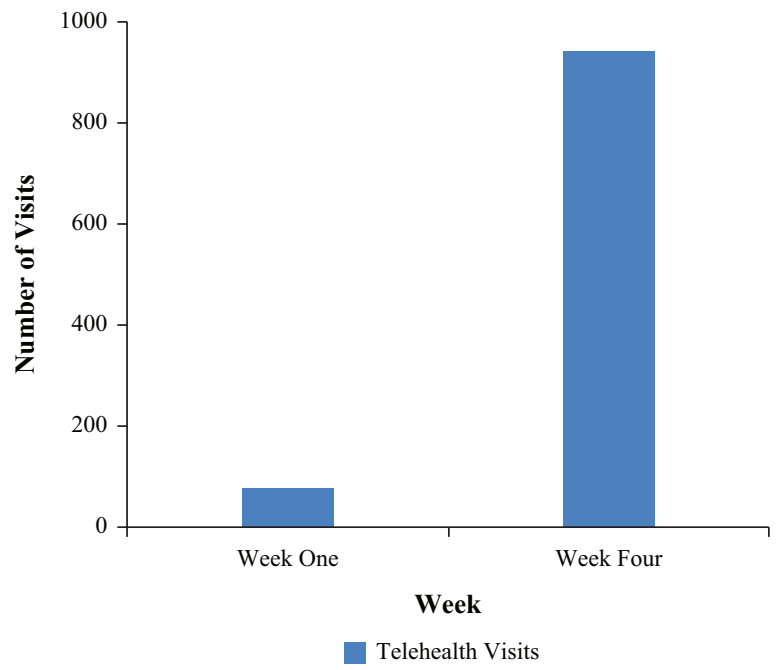

Figure 4-Telehealth ambulatory visits.

been relaxed. However, we believe that the dynamic environment and the uncertainty around the federal government's commitment to sustaining these relaxed regulations remain unclear.

\section{Source of Truth}

With multiple clinics using this solution, we felt that one source of truth was of the utmost importance. This would allow a patient (or provider) to e-mail or call one number to obtain help. Owing to multiple vendors in our telehealth space, we were not able to complete this entirely across the enterprise. We have implemented a service for each of our platforms.

\section{Adoption}

With the very swift culture change, our enterprise suffered from diffusing this idea to the masses of staff, providers, and patients. Our group did see the same trends outlined in Rogers' Diffusion of Innovation Model. ${ }^{5}$ Many of the barriers of adoption were curtailed with continued education and communication.

Our group was able to revise our training plan toward the end to tailor the education material to each specialty and software experience. Having one source of truth in each cohort allowed some wins across the enterprise due to the following:

Individual optimization sessions: Our group created a continuous improvement initiative in an effort to identify providers who had a lower number of completed telehealth visits compared to their peers. The group scheduled one-on-one sessions to discuss adoption barriers. 
Regulatory: West Tennessee Healthcare services has many partial or full healthcare professional shortage areas. The uncertainty in regulation changes and potential pull backs to these regulations do create some questions in the upcoming months. We devised a strategic plan to build as if these changes were rolled back completely.

Role incertitude: Our group identified some role confusion with the nursing and support staff role. The set workflow of nurses rooming the patient, triaging the patient, and obtaining medication and social history — among other tasks — changed relatively quickly with this implementation. This role change was also shared by the support staff with their traditional interactions deviating significantly. We are still working to optimize and craft these roles to allow them to work most efficiently in that role.

Custom content updates and dissemination: Overall, the custom content changes were well received by the clinical and support staff. The clinicians readily adapted to changes provided to them in this very quick implementation exercise. We are continuously working to refine and improve our workflows and communication.

\section{CONCLUSIONS}

Telehealth offered a great opportunity for the clinicians at West Tennessee Healthcare to provide an essential service to the community at large during these unprecedented times. Our telehealth footprint continues to enlarge daily with more clinicians and specialties becoming comfortable with the workflows and technology.

Even though this public health crisis served as a major catalyst for telehealth adoption, it is clear that telehealth is here to stay. We plan to optimize our ambulatory and inpatient solutions to continue to provide high-quality and patient-centered care. In the near future, West Tennessee Healthcare plans to formally survey patients that have used telehealth regarding perception of telehealth, potential gaps, and areas of improvement.

Funding statement: This work was not supported by grants or awards.

Conflict of Interest: Claude J. Pirtle, MD, MSACI, is a member of the content review board of Telehealth and Medicine Today. The authors have no other conflicts of interest.

Contributors: All authors contributed equally to this paper.

\section{REFERENCES}

1. Castellucci M, Meyer H. Physician practices modify operations to cope with COVID-19.

2. Kotter JP. Leading change: Why transformation efforts fail. Harvard Business Review. 1995 March-April, 1-8. Available from: https://hbr. org/1995/05/leading-change-whytransformation-efforts-fail-2.

3. OCR announces notification of enforcement discretion for Telehealth remote communications during the COVID-19 nationwide public health emergency. 17 March 2020. Available from: https://www.hhs.gov/about/ news/2020/03/17/ocr-announcesnotification-of-enforcement-discretionfor-telehealth-remote-communicationsduring-the-covid-19.html.

4. Lorenzi NM, Riley RT. Managing change: An overview. J Am Med Inform Assoc. 2000;7(2):116-24. https://doi.org/10.1136/ jamia.2000.0070116

5. "Diffusion of Innovation Theory." [cited 2020 May 04]. Available from: http://sphweb.bumc.bu.edu/otlt/MPHModules/SB/BehavioralChangeTheories/ BehavioralChangeTheories4.html 
Copyright Ownership: This is an open access article distributed in accordance with the Creative Commons Attribution Non Commercial (CC BY-NC 4.0) license, which permits others to distribute, adapt, enhance this work non-commercially, and license their derivative works on different terms, provided the original work is properly cited and the use is non-commercial. See: http://creativecommons.org/licenses/ by-nc/4.0. 